\title{
DOA Estimation of Multiple LFM Sources Using a STFT-based and FBSS-based MUSIC Algorithm
}

\author{
Kaibo CUI, Xi CHEN, Jingjian HUANG, Naichang YUAN \\ College of Electronic Science and Engineering, National University of Defense Technology \\ Changsha, Hunan, 410073, China \\ 764608294@qq.com
}

Submitted August 22, 2017 / Accepted October 23, 2017

\begin{abstract}
Direction of arrival (DOA) estimation is an important problem in array signal processing. An effective multiple signal classification (MUSIC) method based on the short-time Fourier transform (STFT) and forward/ backward spatial smoothing (FBSS) techniques for the $D O A$ estimation problem of multiple time-frequency ( $t-f)$ joint LFM sources is addressed. Previous work in the area e. g. STFT-MUSIC algorithm cannot resolve the t-f completely or largely joint sources because they can only select the single-source t-f points. The proposed method constructs the spatial t-f distributions (STFDs) by selecting the multiple-source $t$-f points and uses the FBSS techniques to solve the problem of rank loss. In this way, the STFTFBSS-MUSIC algorithm can resolve the t-f largely joint or completely joint LFM sources. In addition, the proposed algorithm also owns pretty low computational complexity when resolving multiple LFM sources because it can reduce the times of the feature decomposition and spectrum search. The performance of the proposed method is compared with that of the existing $t$-f based MUSIC algorithms through computer simulations and the results show its good performance.
\end{abstract}

\section{Keywords}

DOA estimation, STFT, FBSS, LFM signals, t-f joint, MUSIC, multiple-source t-f points

\section{Introduction}

In recent years, the direction of arrival (DOA) estimation has received tremendous attention in different areas, such as radar, sonar and communication [1-5]. Many algorithms, such as multiple signal classification (MUSIC) [6], estimation of signal parameters via rotation invariance techniques (ESPRIT) [7], [8] and their variants based on the Eigen-structure techniques, have been developed over the years and provided satisfactory performance. Nevertheless, the traditional MUSIC-like and ESPRIT-like algorithms have limitations with regard to resolving coherent sources and wideband sources. Linear frequency-modulated (LFM), or chirp, signals are frequently encountered in applications such as radar, sonar, bioengineering, and so forth. So the DOA estimation of LFM signals also has become a hot issue in array signal processing [9-17]. As we know, time-frequency ( $\mathrm{t}-\mathrm{f})$ analysis tools are effective tools to deal with LFM signals because of the concentration property of the signals in the $t-f$ domain, so a series of DOA estimation algorithms based on spatial time-frequency distributions (STFDs) have been proposed [18-24]. These algorithms use the STFD matrices instead of the covariance matrices in the traditional subspace algorithms [6-8]. Compared to the traditional algorithms, the t-f based algorithms can provide better DOA estimation performance since the $\mathrm{t}-\mathrm{f}$ analysis tools spread the noise power while localizing the source energy in the t-f domain. Moreover, the selection of $\mathrm{t}-\mathrm{f}$ points belonging to a specific set of sources allows these algorithms estimate more sources with a small number of sensors [20], [25], [26]. By selecting auto-source $t-f$ points, these algorithms can also solve the DOAs of closely-spaced sources [25-27].

The existing t-f based algorithms mainly use two kinds of assumptions. 1) The sources are disjoint in the t-f plane, which means the sources are completely independent of each other in the $t-f$ domain. So, the $t-f$ disjoint sources do not have the same t-f points in the t-f plane [25], [27-29]. 2) The sources are tiny joint in the t-f domain, which means multiple sources just have very few of the same t-f points in the t-f domain [26], [30], [31]. Under this scenario, auto-terms t-f points [18], [20], [25], [27], [30] or cross-terms t-f points [19] have been considered to construct STFD matrices. Due to the computational complexity and the cross-terms of quadratic t-f analysis tools, the linear t-f analysis tools, e.g., the fractional Fourier transform (FRFT) [10], [13], [15], [16], [32] and the short-time Fourier transform (STFT) [33-35], also have been applied to the DOA estimation field. These algorithms also use the above two assumptions, which means they all cannot resolve the t-f completely or largely joint sources (the t-f points of the sources are all the same or the sources have a lot of the same $t-f$ points), which limits their further developments. 
In order to ease the limitations of the existing t-f based algorithms, including quadratic $\mathrm{t}-\mathrm{f}$ analysis tools and linear t-f analysis tools, herein we propose a short-time Fourier transform based and forward/backward spatial smoothing (FBSS) based MUSIC (STFT-FBSS-MUSIC) algorithm. If the sources are $t-f$ largely or completely joint, it is difficult to select the regions in the t-f domain, where a source exists alone. In our research, the STFT is used because its computational complexity is low and the crossterms are avoided compared to the quadratic t-f analysis tools. The forward/backward spatial smoothing (FBSS) technique is also used in our research, which is a kind of dimension decrease algorithm, aiming at solving the problem of rank loss in the covariance matrix caused by the coherent sources or high correlated sources [36-39]. Instead of selecting the single-source t-f points (i.e., the t-f points where only one source exists), we propose that we can select multiple-source $t-f$ points to estimate DOAs of multiple-source simultaneously. In this way, we can solve the problem caused by the low $\mathrm{t}-\mathrm{f}$ resolution of the linear STFT that appropriate single-source $t-f$ points are difficult to be selected, especially in the case of completely joint or largely joint LFM signals, the single-source t-f points even cannot be selected. In addition, if selecting the multiplesource $t-f$ points, the times of feature decomposition and MUSIC spectrum search can be decreased in the case of multiple sources, which means the computational complexity of the STFT-FBSS-MUSIC algorithm will be lower than the STFT-MUSIC algorithm and other t-f based algorithms. Compared to the existing t-f based MUSIC algorithms, the advantages of the proposed STFT-FBSSMUSIC algorithm are as follows:

- The ability to resolve largely t-f joint LFM sources.

- The ability to resolve completely t-f joint LFM sources.

- The low computational complexity.

The simulation and experimental results verify the effectiveness of the proposed algorithm.

\section{The Received Signal Model}

Consider a uniform linear array (ULA) with $N$ isotropic sensors exposed to $M$ far-field LFM sources $s_{1}(t) s_{2}(t) \ldots s_{M}(t)$ whose DOAs are $\theta_{1}, \theta_{2}, \ldots, \theta_{M}$ respectively. The output of the array, including the signals and noise, can be modeled as [6], [12-15]

$$
\mathbf{x}(t)=\mathbf{y}(t)+\boldsymbol{\eta}(t)=\mathbf{A}(t, \boldsymbol{\theta}) \mathbf{s}(t)+\boldsymbol{\eta}(t)
$$

where $\mathbf{x}(t)=\left[x_{1}(t) x_{2}(t) \ldots x_{N}(t)\right]^{\mathrm{T}}$ is the sensor array output vector, $\mathbf{s}(t)=\left[s_{1}(t) s_{2}(t) \ldots s_{M}(t)\right]^{\mathrm{T}}$ are the LFM sources and $\boldsymbol{\eta}(t)=\left[\begin{array}{lll}\eta_{1}(t) & \eta_{2}(t) \ldots \eta_{N}(t)\end{array}\right]^{\mathrm{T}}$ is the additive white Gaussian noise vector. $[\cdot]^{\mathrm{T}}$ is the transpose operator. $\mathbf{A}(t, \boldsymbol{\theta})=\left[\mathbf{a}\left(t, \theta_{1}\right) \mathbf{a}\left(t, \theta_{2}\right) \ldots \mathbf{a}\left(t, \theta_{M}\right)\right] \quad$ is $\quad$ a $N \times M$ manifold vector matrix [6-8], which is related to the parameters of the ULA and received sources. Each column of $\mathbf{A}(t, \boldsymbol{\theta})$ corresponds to a single arrival and carries a clear bearing. $\mathbf{a}\left(t, \theta_{m}\right)$ is the $N \times 1$ direction vector (steering vector [6]) of the $m$ th $(m=1,2, \ldots, M)$ source at time $t$, which can be given by

$$
\mathbf{a}\left(t, \theta_{m}\right)=\left[\begin{array}{c}
1 \\
\exp \left(-\mathrm{j} 2 \pi f_{m}(t) d \sin \theta_{m} / c\right. \\
\vdots \\
\exp \left(-\mathrm{j} 2 \pi f_{m}(t)(N-1) d \sin \theta_{m} / c\right.
\end{array}\right]
$$

where $d$ denotes the inter-element spacing of the sensors and $c$ is the propagation velocity. $f_{m}(t)$ is the instantaneous frequency of the $m$ th LFM signal at $t$, which can be given by

$$
f_{m}(t)=f_{m}+k_{m} t
$$

where $f_{m}$ and $k_{m}$ denote initial frequency and frequency modulation rate of the $m$ th LFM signal, respectively.

\section{The Proposed STFT-FBSS-MUSIC Algorithm}

\subsection{The Spatial Time-Frequency Distribution Based on STFT}

The STFT of the array output $\mathbf{x}(t)$ in (1) is computed as $[33,35]$

$$
\mathbf{S}_{\mathbf{x}}(t, f)=\operatorname{STFT}(\mathbf{x}(t))=\mathbf{A}(t, \theta) \mathbf{S}_{\mathbf{S}}(t, f)+\mathbf{S}_{\boldsymbol{\eta}}(t, f)
$$

where $\mathbf{S}_{\mathbf{x}}(t, f)=\left[\mathbf{S}_{x_{1}}(t, f) \mathbf{S}_{x_{2}}(t, f) \ldots \mathbf{S}_{x_{N}}(t, f)\right]^{\mathrm{T}}$ and $\mathbf{S}_{x_{n}}(t, f)$ denotes the STFT value of the $n$th sensor output. $\mathbf{S}_{\mathbf{s}}(t, f)=\left[\mathbf{S}_{s_{1}}(t, f) \mathbf{S}_{s_{2}}(t, f) \ldots \mathbf{S}_{s_{M}}(t, f)\right]^{\mathrm{T}}$ and $\mathbf{S}_{s_{m}}(t, f)$ denotes the STFT value of the $m$ th source. $\mathbf{S}_{\boldsymbol{\eta}}(t, f)=\left[\mathbf{S}_{\eta_{1}}(t, f) \mathbf{S}_{\eta_{2}}(t, f) \ldots \mathbf{S}_{\eta_{N}}(t, f)\right]^{\mathrm{T}}$ and $\mathbf{S}_{\eta_{n}}(t, f)$ denotes the STFT value of the $n$th sensor's noise.

Define $\mathbf{D}_{\mathbf{x x}}(t, f)$ as the STFD matrix of the array output based on STFT [18-20], [35], which can be formulated as

$$
\begin{aligned}
& \mathbf{D}_{\mathbf{x} x}(t, f)=\mathbf{S}_{\mathbf{x}}(t, f) \mathbf{S}_{\mathbf{x}}^{\mathrm{H}}(t, f) \\
& =\left[\begin{array}{ccc}
\mathbf{S}_{x_{1}}(t, f) \mathbf{S}_{x_{1}}^{*}(t, f) & \cdots & \mathbf{S}_{x_{1}}(t, f) \mathbf{S}_{x_{N}}^{*}(t, f) \\
\vdots & \ddots & \vdots \\
\mathbf{S}_{x_{N}}(t, f) \mathbf{S}_{x_{1}}^{*}(t, f) & \cdots & \mathbf{S}_{x_{N}}(t, f) \mathbf{S}_{x_{N}}^{*}(t, f)
\end{array}\right] \\
& =\mathbf{A}(t, \theta) \mathbf{S}_{\mathbf{s}}(t, f) \mathbf{S}_{\mathbf{s}}^{\mathrm{H}}(t, f) \mathbf{A}(t, \theta)+\mathbf{A}(t, \theta) \mathbf{S}_{\mathbf{s}}(t, f) \mathbf{S}_{\boldsymbol{\eta}}^{H}(t, f)+ \\
& \mathbf{S}_{\boldsymbol{\eta}}(t, f) \mathbf{S}_{\mathbf{s}}^{\mathrm{H}}(t, f) \mathbf{A}^{\mathrm{H}}(t, \theta)+\mathbf{S}_{\boldsymbol{\eta}}(t, f) \mathbf{S}_{\boldsymbol{\eta}}^{\mathrm{H}}(t, f) \\
& =\mathbf{A}(t, \theta) \mathbf{D}_{\mathbf{s s}}(t, f) \mathbf{A}(t, \theta)+\mathbf{A}(t, \theta) \mathbf{S}_{\mathbf{s}}(t, f) \mathbf{S}_{\boldsymbol{\eta}}^{\mathrm{H}}(t, f)+ \\
& \mathbf{S}_{\boldsymbol{\eta}}(t, f) \mathbf{S}_{\mathbf{s}}^{\mathrm{H}}(t, f) \mathbf{A}^{\mathrm{H}}(t, \theta)+\mathbf{D}_{\boldsymbol{\eta} \boldsymbol{\eta}}(t, f)
\end{aligned}
$$

where the superscript $[\cdot]^{\mathrm{H}}$ denotes the complex conjugate transpose of a matrix and $[\cdot]^{*}$ is the conjugate operator. 
$\mathbf{D}_{\mathbf{S S}}(t, f)$ and $\mathbf{D}_{\eta \eta}(t, f)$ respectively denote the STFD matrix of the source and noise.

As the noise is the additive white Gaussian noise, so there are $\mathrm{E}\left[\mathbf{A}(t, \boldsymbol{\theta}) \mathbf{S}_{\mathbf{s}}(t, f) \mathbf{S}_{\eta}{ }^{\mathrm{H}}(t, f)\right]=0 \quad$ and $\mathrm{E}\left[\mathbf{S}_{\boldsymbol{\eta}}(t, \boldsymbol{\theta}) \mathbf{S}_{\mathbf{s}}{ }^{\mathrm{H}}(t, f) \mathbf{A}^{\mathrm{H}}(t, f)\right]=0$, in which $\mathrm{E}[\cdot]$ denotes the expectation operator. As a result, equation (5) can be written as

$$
\mathrm{E}\left[\mathbf{D}_{\mathbf{x x}}(t, f)\right]=\mathbf{A}(t, \theta) \mathbf{D}_{\mathbf{s s}}(t, f) \mathbf{A}(t, \theta)+\mathrm{E}\left[\mathbf{D}_{\boldsymbol{\eta} \boldsymbol{\eta}}(t, f)\right] .
$$

Equation (6) is similar to the formula that has been commonly used in DOA estimation, relating the signal correlation matrix to the data spatial correlation matrix except for the correlation matrices replaced by the STFD matrices. The well-established results in conventional DOA estimation field [6-8] (i.e. MUSIC) could, therefore, be utilized in this situation, which means we can use the STFD matrix to estimate DOAs of LFM signals.

It is noted that (6) holds true for every t-f point in the t-f plane. In the t-f DOA estimation field, the algorithm always makes the best of reducing the effect of noise and ensuring the full column rank property of the STFD matrix. Joint diagonalization [31], [40], [41] and t-f averaging [20], [27] are two main approaches that have been used for this purpose. In this paper, however, we only consider averaging over multiple $t-f$ points. Assuming a group of $t-f$ points in the t-f plane are determined, the averaged STFD matrix are obtained by [35]

$$
\mathbf{D}=\frac{1}{L_{0}} \sum_{i=1}^{L_{0}} \mathbf{D}_{\mathbf{x x}}\left(t_{i}, f_{i}\right)
$$

where $\left(t_{i}, f_{i}\right)$ denotes the selected t-f point and $L_{0}$ is the number of the picked out $t-f$ points.

\subsection{The Forward/Backward Spatial Smoothing Processing Technique}

As discussed in Sec. 1, if the LFM sources are completely joint or largely joint in the t-f plane, it is difficult to select the single-source $t-f$ points in the $t-f$ domain, which means the t-f points in (7) might contain the multiplesource $t-f$ points. With regard to the multiple-source $t-f$ points, they denote that different sources own the same frequency at these t-f points, which means the far-field sources become the coherent signals. As is well-known, coherent signals can cause rank deficiency in the correlation matrix of received signals [36]. So coherent LFM signals can also cause rank deficiency in the STFD matrices. People have developed a series of algorithms to overcome the coherency problem [36-39], [42], [43], e.g., the forward/backward spatial smoothing (FBSS) technique [37] and the Toeplitz methods [42], [43]. In this paper, we use the FBSS technique to overcome the rank deficiency in the STFD matrices caused by the multiple-source t- $f$ points. The FBSS technique is a kind of dimension decrease algorithm, which segments the array into several overlapped sub-arrays and then utilizes the average of the STFDs matrices of sub-arrays to solve the rank deficiency problem.

Assuming the ULA is divided into $p$ overlapping forward sub-arrays and each sub-array owns $k$ sensors, which means $N=p+k-1$. Let $\mathbf{x}_{i}^{f}(t)$ stand for the output of the $i$ th sub-array for $i=1,2, \ldots, p$. Using (1), (2), we can get the sub-array model without noise

$$
\begin{aligned}
& \mathbf{x}_{i}^{f}(t)=\left[\begin{array}{llll}
x_{i}(t) & x_{i+1}(t) & \cdots & x_{i+k-1}(t)
\end{array}\right]^{\mathrm{T}} \\
& =\mathbf{A}(t, \boldsymbol{\theta}) \mathbf{B}^{i-1}(t) \mathbf{s}(t), 1 \leq i \leq p, \\
& \mathbf{B}(t)=\operatorname{diag}\left\{\exp \left(\mathrm{j} \beta_{1}(t)\right) \quad \cdots \quad \exp \left(\mathrm{j} \beta_{M_{0}}(t)\right)\right\}, \\
& \beta_{m}(t)=2 \pi d f_{m}(t) \sin \theta_{m} / \mathrm{c}, \quad m=1,2, \ldots, M_{0}
\end{aligned}
$$

where $M_{0}$ denotes the number of sources selected out from $M$ sources based on the selected multiple-source t-f points.

So, the STFD matrix of the $i$ th sub-array can be given by

$$
\mathbf{D}_{i}^{f}=\mathbf{A}(t, \boldsymbol{\theta}) \mathbf{B}^{i-1}(t) \mathbf{D}_{\mathbf{s s}}(t, f)\left(\mathbf{B}^{i-1}(t)\right){ }^{\mathrm{H}} \mathbf{A}^{\mathrm{H}}(t, \boldsymbol{\theta}) .
$$

The forward spatially smoothed STFD matrix $\mathbf{D}^{f}$ is the mean of the forward sub-array STFDs matrices, so

$$
\begin{gathered}
\mathbf{D}^{f}=\frac{1}{p} \sum_{i=1}^{p} \mathbf{D}_{i}^{f}=\mathbf{A}(t, \boldsymbol{\theta}) \mathbf{D}_{\mathbf{s s}}^{f}(t, f) \mathbf{A}^{\mathrm{H}}(t, \boldsymbol{\theta}), \\
\mathbf{D}_{\mathbf{s s}}^{f}(t, f)=\frac{1}{p} \sum_{i=1}^{p} \mathbf{B}^{i-1}(t) \mathbf{D}_{\mathbf{s s}}(t, f)\left(\mathbf{B}^{i-1}(t)\right)^{\mathrm{H}} .
\end{gathered}
$$

Additional $p$ backward sub-arrays are generated from the same set of sensors by grouping elements at $\{N, N-1$, $\ldots, N-k+1\}$ to form the first backward sub-array and elements at $\{N-1, N-2, \ldots, N-k\}$ to form the second one, etc. Let $\mathbf{x}_{i}^{b}(t)$ represent the complex conjugate of the out of the $i$ th backward sub-array for $i=1,2, \ldots, p$. So, we have

$$
\mathbf{x}_{i}^{b}(t)=\left[\begin{array}{llll}
x_{N-i+1}^{*}(t) & x_{N-i}^{*}(t) & \cdots & x_{N-k-i+2}^{*}(t)
\end{array}\right]^{\mathrm{T}} .
$$

The $i$ th forward sub-array and the $(p-i+1)$ th backward sub-array have the following relationship

$$
\begin{aligned}
& \mathbf{x}_{p-i+1}^{b}(t)=\mathbf{J}\left(\mathbf{x}_{p}^{f}(t)\right){ }^{*}=\mathbf{J} \mathbf{A}^{*}(t, \boldsymbol{\theta}) \mathbf{B}^{-(p-1)}(t) \mathbf{s}^{*}(t),
\end{aligned}
$$

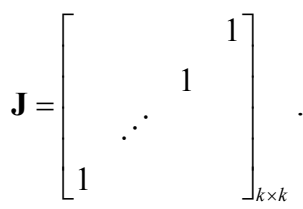

Then, the STFDs matrix of the $(p-i+1)$ th backward sub-array is given by:

$\mathbf{D}_{p-i+1}^{b}=$

$\mathbf{J A}^{*}(t, \boldsymbol{\theta}) \mathbf{B}^{-(i-1)}(t) \mathbf{D}_{\mathbf{s s}}^{*}(t, f) \cdot\left(\mathbf{B}^{-(i-1)}(t)\right)^{\mathrm{H}}\left(\mathbf{A}^{\mathrm{H}}(t, \boldsymbol{\theta}) \mathbf{J}\right){ }^{*}$. 
Using (16), we can get $\mathbf{J}=\mathbf{J}^{*}=\mathbf{J}^{\mathrm{T}}=\mathbf{J}^{\mathrm{H}}$, so $\left(\mathbf{A}^{\mathrm{H}}(t, \boldsymbol{\theta}) \mathbf{J}\right)^{*}=\left(\mathbf{J A}^{*}(t, \boldsymbol{\theta})\right)^{\mathrm{H}}$ and $\mathbf{J} \mathbf{A}^{*}(t, \boldsymbol{\theta})=\mathbf{A}(t, \boldsymbol{\theta}) \mathbf{B}^{k-1}(t)$. Then we can get

$$
\mathbf{D}_{p-i+1}^{b}=\mathbf{A}(t, \boldsymbol{\theta}) \mathbf{B}^{k-i}(t) \mathbf{D}_{\mathbf{s s}}^{*}(t, f) \mathbf{B}^{i-k}(t) \mathbf{A}^{\mathrm{H}}(t, \boldsymbol{\theta}) .
$$

The backward spatially smoothed STFDs matrix $\mathbf{D}^{b}$ is the mean of the backward sub-array STFDs matrices, so

$$
\begin{gathered}
\mathbf{D}^{b}=\frac{1}{p} \sum_{i=1}^{p} \mathbf{D}_{p-i+1}^{b}=\mathbf{A}(t, \boldsymbol{\theta}) \mathbf{D}_{\mathbf{s s}}^{b}(t, f) \mathbf{A}^{\mathrm{H}}(t, \boldsymbol{\theta}), \\
\mathbf{D}_{\mathbf{s s}}^{b}(t, f)=\frac{1}{p} \sum_{i=1}^{p} \mathbf{B}^{k-i}(t) \mathbf{D}_{\mathbf{s s}}^{*}(t, f) \mathbf{B}^{i-k}(t) .
\end{gathered}
$$

According to [37], $\mathbf{D}^{f}$ and $\mathbf{D}^{b}$ is of full rank if $k \geq M_{0}$, $p \geq M_{0}$. So, we can apply the Eigen-structure methods to this smoothed STFDs matrix to estimate the DOAs of LFM sources if selecting the multiple-source t-f points. Following [36], [37], the use of forward and backward sub-array averaging schemes can reduce the number of extra sensors. So, define the forward/backward smoothed STFDs matrix $\mathbf{D}^{f b}$ as the mean of $\mathbf{D}^{f}$ and $\mathbf{D}^{b}$; i.e.,

$$
\mathbf{D}^{f b}=\left(\mathbf{D}^{f}+\mathbf{D}^{b}\right) / 2 .
$$

According to [37], $\mathbf{D}^{f b}$ is of full rank if $k \geq M_{0}$, $2 p \geq M_{0}$. Again, recalling that in presence of $M_{0}$ LFM sources, the size $k$ of each sub-array must be at least $\left(M_{0}+1\right)$, it follows that the number of sensors $N$ must satisfy $N \geq 3 M_{0} / 2$.

Since $\mathbf{D}^{f b}$ has exactly the same form as the traditional STFDs matrix, the Eigen-structure-based techniques [6-8] can be applied to this smoothed STFDs matrix, irrespective of the multiple-source $t-f$ points or the single-source $t-f$ points, to successfully estimate their directions of arrival. Based on this, we can propose the STFT-FBSS-MUSIC algorithm, which estimates the DOA by determining the $M_{0}$ peaks of the spatial spectrum [6]

$$
P(\theta)=\frac{\mathbf{a}^{\mathrm{H}}(\theta) \mathbf{a}(\theta)}{\mathbf{a}^{\mathrm{H}}(\theta) \mathbf{U}_{n} \mathbf{U}_{n}^{\mathrm{H}} \mathbf{a}(\theta)}
$$

where $\mathbf{U}_{n}$ denotes the noise eigenvectors of the forward/ backward smoothed STFDs matrix $\mathbf{D}^{f b}$ in (21), which replaced by the covariance matrix of $\mathbf{x}(t)$ in the conventional MUSIC algorithm [6] and the STFDs matrix in the traditional t-f based MUSIC algorithm [9, 20].

\subsection{Summary}

For the readers' convenience, the step by step procedure of the STFT-FBSS-MUSIC algorithm is given as follows:

1) The STFT of the array output is obtained based on (4);

2) The STFD matrix of the array output based on STFT can be obtained according to (5);
3) Select t-f points (single-source points or multiplesource points) and get the averaged STFD matrix based on (7);

4) Use proper $k$ and $p$ to divide the ULA;

5) Obtain the forward smoothed STFDs matrix and the backward smoothed STFDs matrix based on (12) and (19);

6) Get the forward/backward smoothed STFDs matrix based on (21);

7) Use the MUSIC algorithm to estimate the DOAs of the sources based on (22).

It is noted that the premise of implementing the STFT-FBSS-MUSIC algorithm lies in the available multiple-source $t-f$ points or single-source $t-f$ points sets to construct the forward/backward smoothed STFDs matrix in (21). The t-f points of sources can be separately used for implementing the STFT-FBSS-MUSIC algorithm, which offers the potential ability to estimate the DOAs of LFM signals with a smaller number of sensors, i.e., $M>N$. As discussed above, if selecting the multiple-source t-f points, the number of sources involved in these points must satisfy $M_{0} \geq 2 N / 3$.

\section{Simulation}

In this section, the performance of the proposed method is evaluated and compared against existing $t-f$ based DOA estimation algorithms through simulation examples. In the simulations, we separately choose quadratic t-f analysis tools [18-31] and the linear t-f analysis tools [10], [13], [15], [16], [32] to estimate the DOAs of the LFM sources. In order to restrain the cross-terms of the quadratic $t-f$ analysis tools, the smoothed pseudo WignerVille distribution (SPWVD) is considered in this paper [20]. The FRFT is selected as a linear t-f analysis tool [32]. In addition, the FBSS technique is also combined with the SPWVD and FRFT according to Sec. 3, which is in favor of contrasting the proposed STFT-FBSS-MUSIC algorithm and other t-f based algorithms. A linear array of 8 sensors with equal element spacing of 0.5 wavelength at $f_{\max }\left(f_{\max }\right.$ denotes the maximum value of the LFM signals frequency) is used. The sampling frequency of the system is $360 \mathrm{MHz}$ and the snapshots' number is 512. In the FBSS process, all the algorithms use $p=4, k=5$.

\subsection{T-f Disjoint Sources}

Let us consider three t-f disjoint LFM sources impinging on the ULA from $-20^{\circ}, 0^{\circ}$ and $20^{\circ}$. The frequency distributions of these three sources separately are $[25,50] \mathrm{MHz}, \quad[65,95] \mathrm{MHz}$ and $[120,160] \mathrm{MHz}$. Figure 1 shows the SPWVD, FRFT and STFT of the t-f disjoint LFM sources at $10 \mathrm{~dB}$ SNR and Figure 2 shows the estimation results of these three t-f disjoint LFM sources using multiple algorithms. 


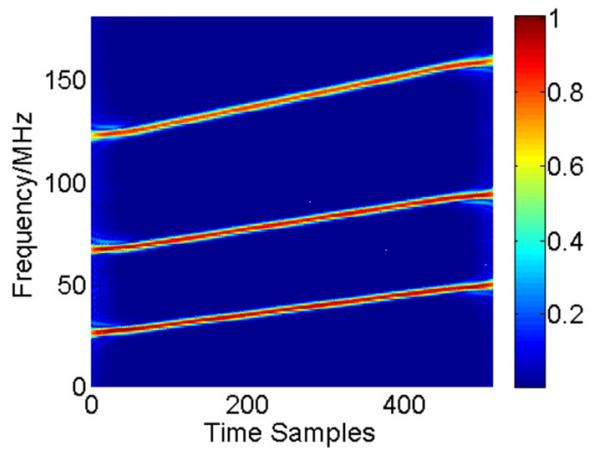

(a)

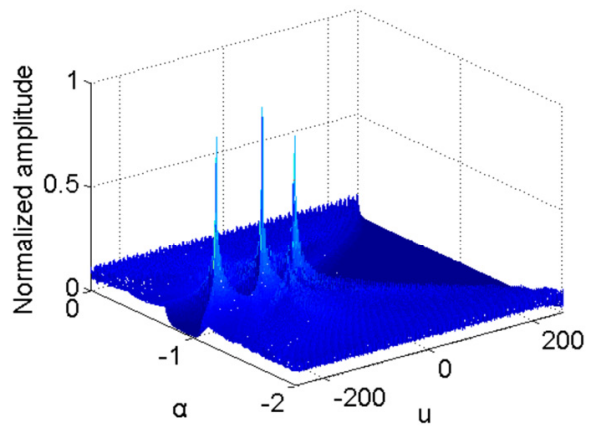

(b)

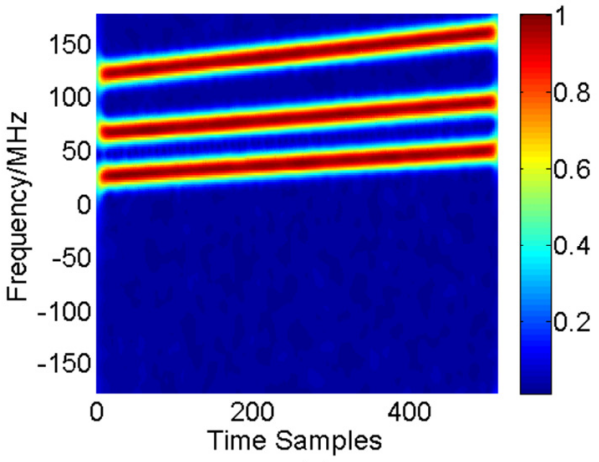

(c)

Fig. 1. (a) SPWVD, (b) FRFT, (c) STFT of the t-f disjoint LFM sources at $10 \mathrm{~dB}$ SNR.

The spatial MUSIC spectrum (see Fig. 2(a)) indicates that there are three sources because we can see three sharp peaks around the correct DOAs for all the algorithms. When using the FBSS technique, we can see that the spatial MUSIC spectrum has a higher energy around the correct DOAs, however, the noise is also elevated. In Fig. 2(b), for comparison purpose, the Cramer-Rao lower bound (CRLB) [44], [45] is also presented. In the next simulation, we present all the CRLB in the RMSE results for the purpose of comparison. It can be seen from the results shown in Fig. 2(b), when estimating the DOAs of t-f disjoint LFM signals, the estimation accuracy of the t-f based FBSSMUSIC algorithms is slightly lower than that of the original $\mathrm{t}-\mathrm{f}$ based MUSIC algorithms. This is because the FBSS technique is a kind of dimension reduction algorithm, which makes the loss of the array aperture. From the results, we can also see that the STFT-based MUSIC algorithms have the best estimation performance. In the case of low SNR, the TF-based MUSIC algorithms and FRFT-

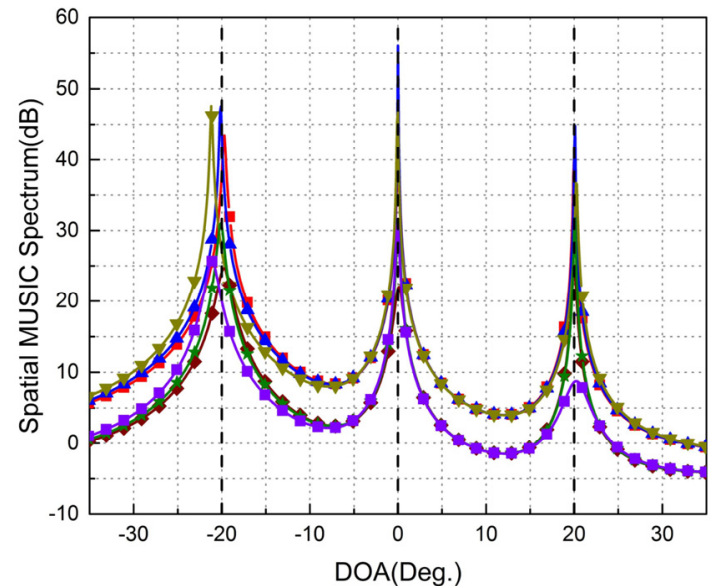

(a)

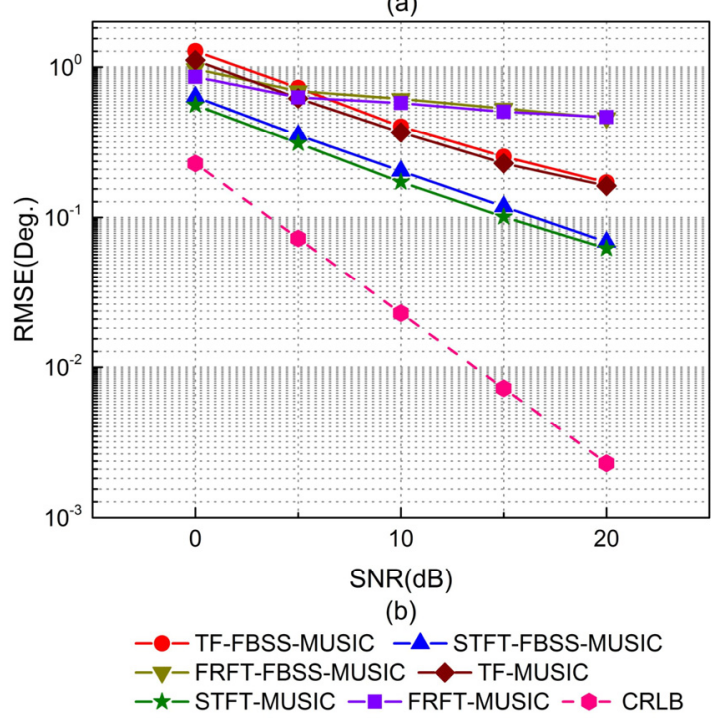

Fig. 2. The estimation results of three $t-f$ disjoint LFM sources using multiple algorithms. (a) The spatial MUSIC spectrum at $10 \mathrm{~dB}$ SNR. (b) The estimated RMSE versus different SNR levels.

based MUSIC algorithms have comparative estimation performance. With the increase of SNR, the estimation accuracy of the FRFT-based MUSIC algorithms is much lower than that of the TF-based MUSIC algorithms. In order to restrain the cross-terms, we choose SPWVD as the quadratic $t-f$ analysis tool which brings about the lower estimation accuracy of the TF-based MUSIC algorithms compared with the STFT-based MUSIC algorithms. In this case, the selected t-f points are definitely single-source t-f points. If there are $M \mathrm{t}-\mathrm{f}$ disjoint LFM sources from farfield, these algorithms need $M$ times feature decomposition and $M$ MUSIC spectrum search [6].

\subsection{T-f Tiny Joint Sources}

Let us consider three t-f tiny disjoint LFM sources impinging on the ULA from $-20^{\circ}, 0^{\circ}$ and $20^{\circ}$. The frequency distributions of these three sources separately are $[10,90] \mathrm{MHz},[40,150] \mathrm{MHz}$ and $[140,10] \mathrm{MHz}$. Figure 3 shows the SPWVD, FRFT and STFT of the t-f tiny disjoint LFM sources at $10 \mathrm{~dB}$ SNR and Figure 4 shows the estimation results of these three sources using multiple algorithms. 


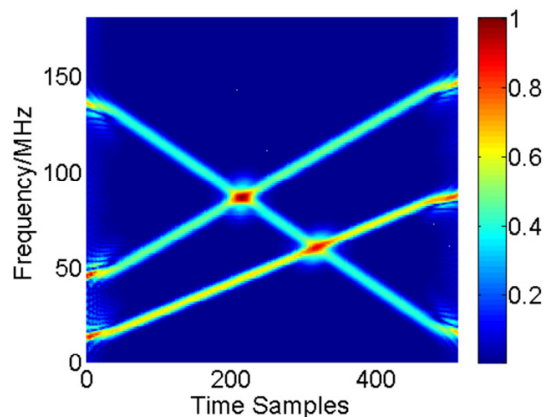

(a)

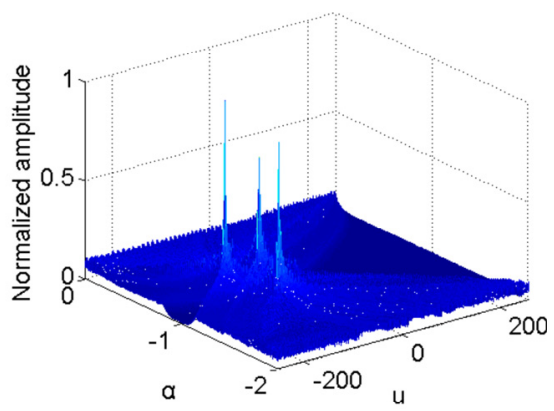

(b)

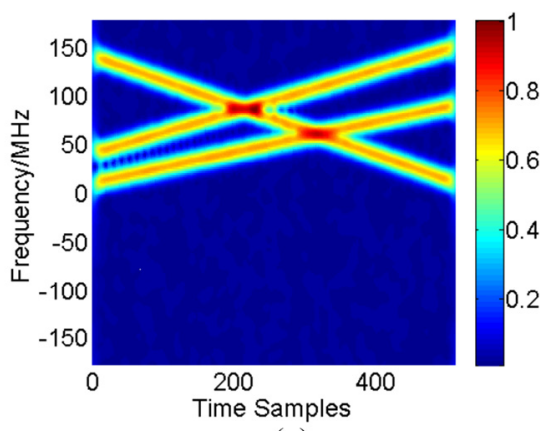

(c)

Fig. 3. (a) SPWVD, (b) FRFT, (c) STFT of the t-f tiny joint LFM sources at $10 \mathrm{~dB}$ SNR.

The spatial MUSIC spectrum (see Fig. 4(a)) illustrates that these algorithms can estimate the DOAs of the t-f tiny joint sources because we can see three sharp peaks around the correct DOAs for all the algorithms. It can be seen from the estimation RMSE curves (see Fig. 4(b)) that the performance of these algorithms is reduced if the LFM sources are $t-f$ tiny joint compared to the $t-f$ disjoint LFM sources. Especially for the FRFT-based MUSIC algorithms [10], [13], [15], [16], [32], in the case of high sources SNR, its estimation RMSE is still more than $1^{\circ}$. The simulation results also show the estimation performance of the STFTFBSS-MUSIC algorithm and the TF-FBSS-MUSIC algorithm if they select the multiple-source t-f points. As can be seen from the results that they also have high DOA estimation accuracy, however, their estimation performance is slightly declined compared to the algorithms selecting the single-source t-f points. In addition, if selecting the multiple-source t-f points, the computational complexity of the STFT-FBSS-MUSIC algorithm and TF-FBSS-MUSIC algorithm can be reduced obviously. As described above, if there are $M$ LFM sources, when selecting the single-source t-f points, the algorithms need $M$ times feature decomposi-

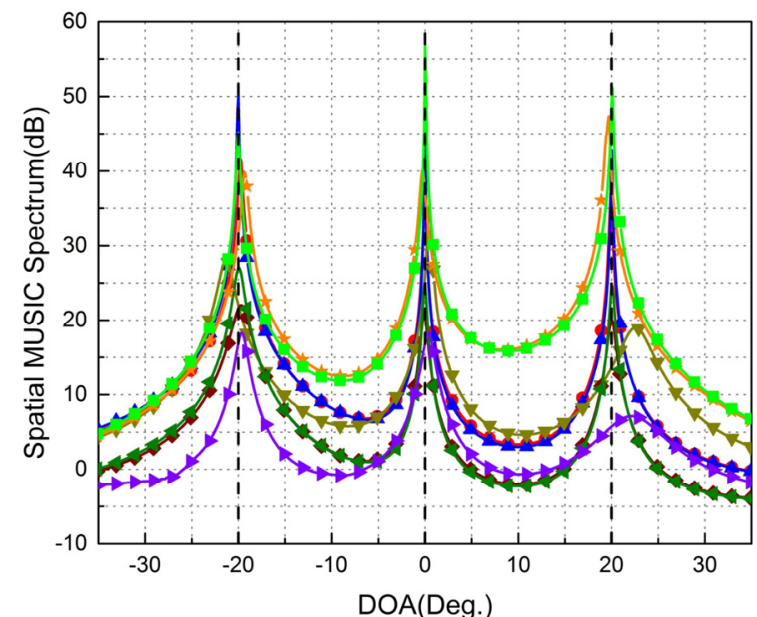

(a)

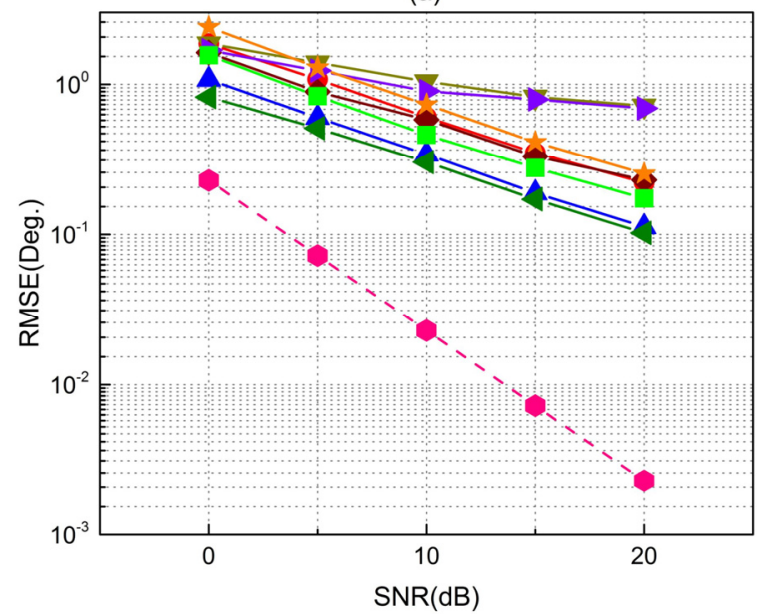

(b)

-O-TF-FBSS-MUSIC - $\mathbf{A}$-STFT-FBSS-MUSIC $-\checkmark-$ FRFT-FBSS-MUSIC - -STFT-MUSIC

- FRFT-MUSIC - TF-MUSIC - -CRLB $-\star-T F-F B S S-M U S I C$ with multiple-source $t-f$ points $-\square-S T F T-F B S S-M U S I C$ with multiple-source t-f points

Fig. 4. The estimation results of three $t-f$ tiny joint LFM sources using multiple algorithms. (a) The spatial MUSIC spectrum at $10 \mathrm{~dB}$ SNR. (b) The estimated RMSE versus different SNR levels.

tion and MUSIC spectrum search, however, if selecting the multiple-source t-f points, the algorithms just need one time feature decomposition and MUSIC spectrum search no matter how many sources are involved in these multiple-source t-f points.

\subsection{T-f Largely Joint Sources}

Let us consider three t-f largely joint LFM sources impinging on the ULA from $-20^{\circ}, 0^{\circ}$ and $20^{\circ}$. The frequency distributions of these three sources separately are $[60,120] \mathrm{MHz},[40,100] \mathrm{MHz}$ and $[50,110] \mathrm{MHz}$. Figure 5 shows the SPWVD, FRFT and STFT of the t-f largely joint LFM sources at $10 \mathrm{~dB}$ SNR and Figure 6 shows the estimation results of these three sources using multiple algorithms.

It can be seen from the results shown in Fig. 5, when the LFM sources are t-f largely joint, the single-source t-f 


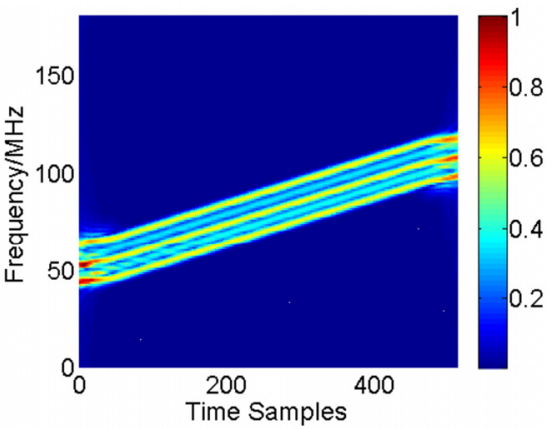

(a)

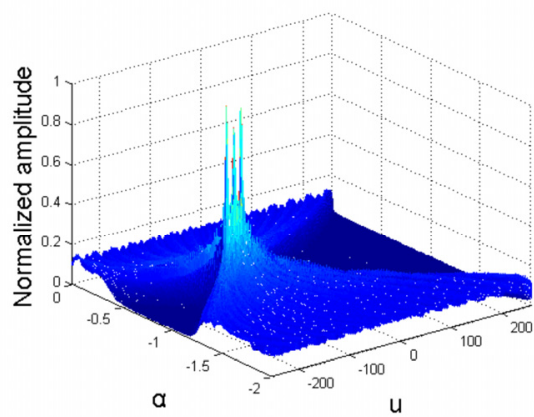

(b)

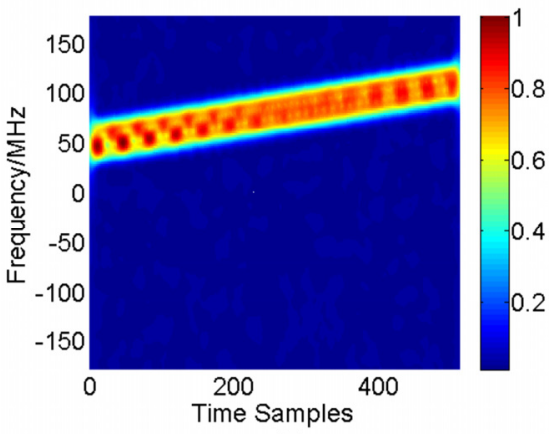

(c)

Fig. 5. (a) SPWVD, (b) FRFT, (c) STFT of the t-f largely joint LFM sources at $10 \mathrm{~dB}$ SNR.

points are pretty difficult to be selected or are not able to be selected by using the SPWVD or STFT. The FRFT can make the LFM sources show three close peak points in the fractional Fourier domain, so we can still separate the peak points for DOA estimation. The MUSIC spectrum of the algorithms shown in Fig. 6-(a) shows that they can resolve the $t-f$ largely joint LFM sources. However, the estimation performance of the FRFT-based algorithms has been very powerful. In the case of low SNR, the estimation accuracy of the TF-FBSS-MUSIC algorithm and STFT-FBSSMUSIC algorithm is low, however, with the increase of SNR, the estimation RMSEs of these two algorithms decrease rapidly.

\subsection{T-f Completely Joint Sources}

Let us consider three t-f completely joint LFM sources impinging on the ULA from $-20^{\circ}, 0^{\circ}$ and $20^{\circ}$. The

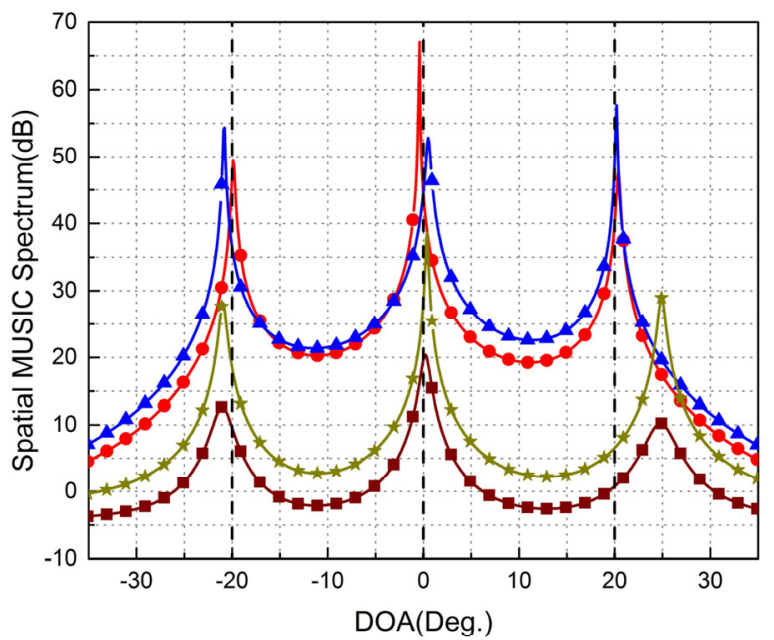

(a)

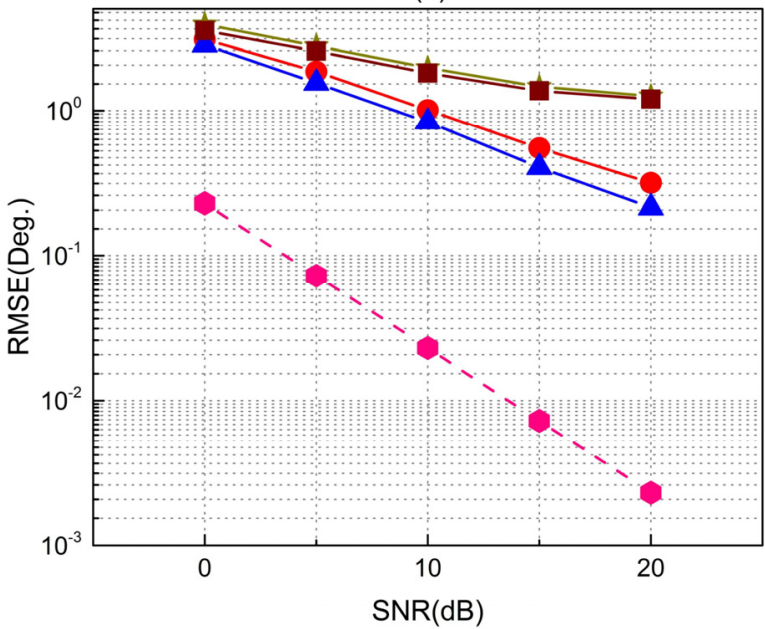

(b)

- TF-FBSS-MUSIC with multiple-source t-f points - $\mathbf{A}$-STFT-FBSS-MUSIC with multiple-source t-f points $-\star-F R F T-F B S S-M U S I C-\square-F R F T-M U S I C-\square-$ CRLB

Fig. 6. The estimation results of three $t-f$ largely joint LFM sources using multiple algorithms. (a) The spatial MUSIC spectrum at $10 \mathrm{~dB}$ SNR. (b) The estimated RMSE versus different SNR levels.

frequency distributions of these three sources are all $[60,120] \mathrm{MHz}$. Figure 7 shows the SPWVD, FRFT and STFT of the t-f completely joint LFM sources at $10 \mathrm{~dB}$ SNR and Figure 8 shows the estimation results of these three sources using multiple algorithms.

As can be seen from the results shown in Fig. 7, when the LFM sources are t-f completely joint, they are completely coherent sources and these three t-f analysis tools cannot resolve the sources in the t-f plane, which means all of the $t-f$ points in the $t-f$ plane are the multiple-source $t-f$ points. Figure 8(a) shows that the spatial MUSIC spectrum of the FRFT-FBSS-MUSIC algorithm has been unable to display the peaks around the correct DOA position, which means it cannot resolve the $t-f$ completely joint LFM sources. The STFT-FBSS-MUSIC algorithm and the TFFBSS-MUSIC algorithm can still work in this case and have pretty high estimation accuracy. 


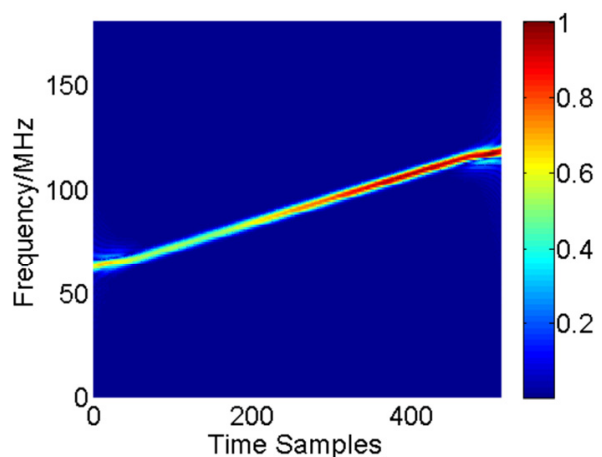

(a)

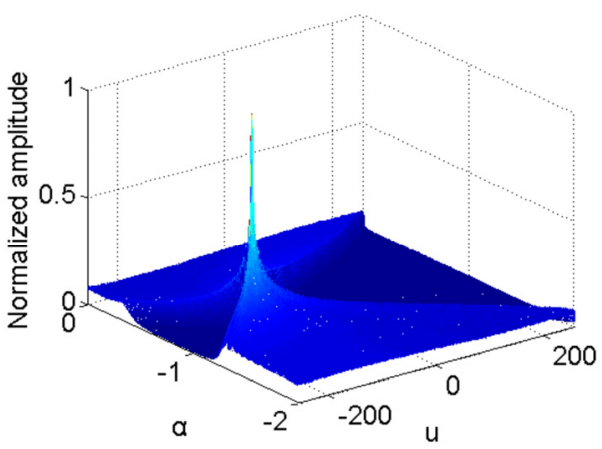

(b)

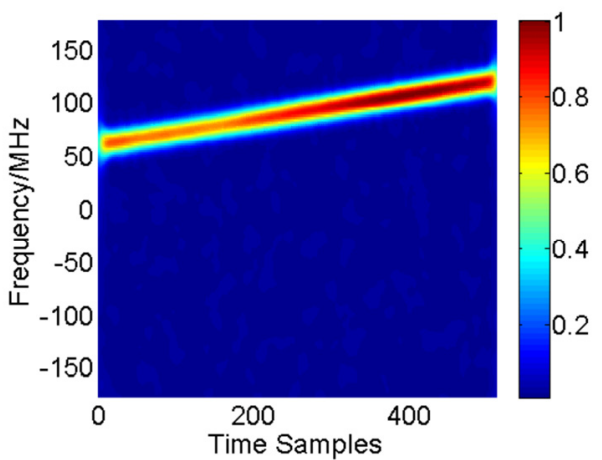

(c)

Fig. 7. (a) SPWVD, (b) FRFT, (c) STFT of the t-f completely joint LFM sources at $10 \mathrm{~dB}$ SNR.

\subsection{Multiple Kinds of Sources using the Proposed Algorithm}

Let us consider nine LFM sources impinging on the ULA from $-40^{\circ},-10^{\circ}, 18^{\circ}, 23^{\circ},-38^{\circ},-20^{\circ},-10^{\circ}, 20^{\circ}$, and $-22^{\circ}$. The frequency distributions of these sources separately are $[10,60] \mathrm{MHz},[10,60] \mathrm{MHz},[10,60] \mathrm{MHz}$, $[150,30] \mathrm{MHz}, \quad[120,70] \mathrm{MHz}, \quad[20,100] \mathrm{MHz}$, $[40,140] \mathrm{MHz},[50,110] \mathrm{MHz}$ and $[165,165] \mathrm{MHz}$. As can be seen from the parameters of the LFM sources, source 1 , source 2 and source 3 are t-f completely joint sources. Source 4 , source 5 and source 6 are t-f tiny joint sources. Source 7 and source 8 are t-f largely joint sources. Source 9 is the t-f disjoint source. Figure 9 shows the estimation results by using the proposed STFT-FBSSMUSIC algorithm.

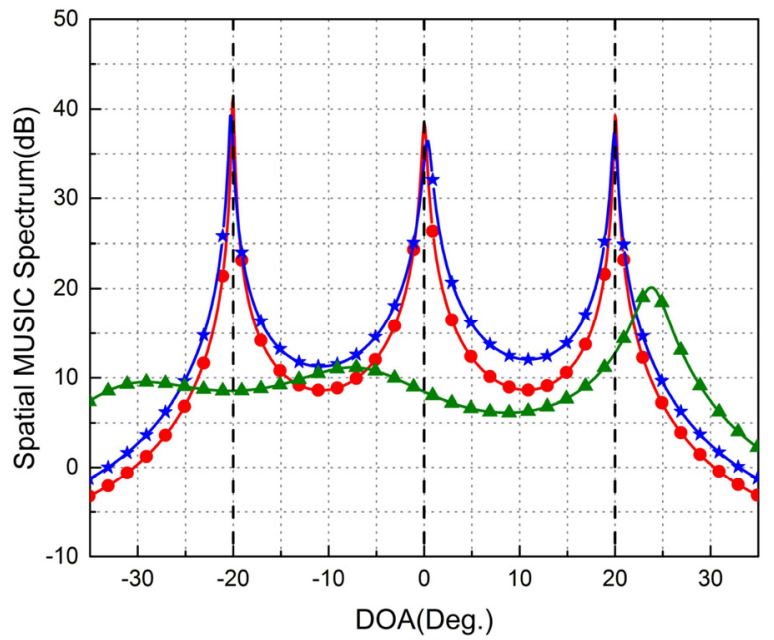

(a)

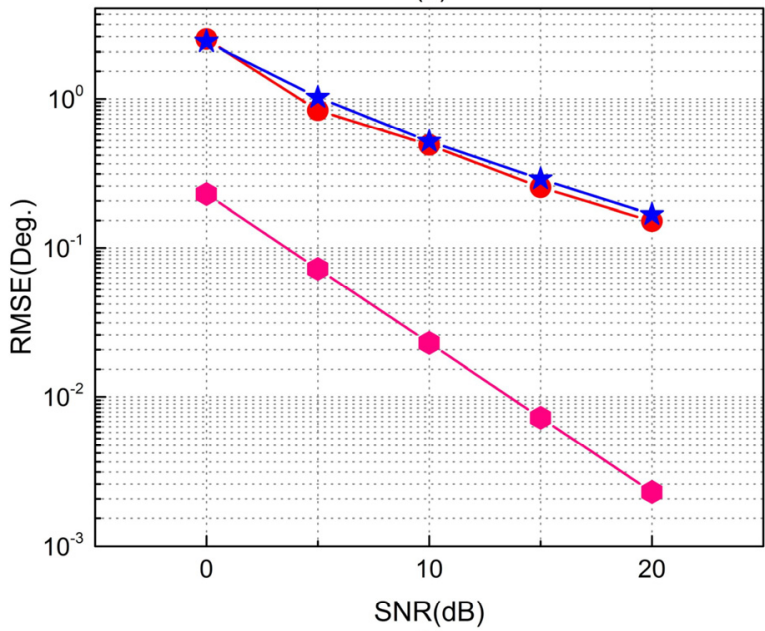

(b)

- TF-FBSS-MUSIC with multiple-source t-f points - $\star$-STFT-FBSS-MUSIC with multiple-source $t-f$ points -A-FRFT-FBSS-MUSIC - - CRLB

Fig. 8. The estimation results of three $t-f$ completely joint LFM sources using multiple algorithms. (a) The spatial MUSIC spectrum at $10 \mathrm{~dB}$ SNR. (b) The estimated RMSE versus different SNR levels.

As can be seen from the STFT of the LFM sources (see Fig. 9(a)), except for the source 9, all the LFM sources are t-f joint (tiny, largely, completely), so we can use STFT-FBSS-MUSIC algorithm to estimate the DOAs of these sources. As can be seen from the results, we can see the STFT-FBSS-MUSIC algorithm not only can resolve multiple kinds of LFM sources, which contain t-f disjoint sources, t-f tiny joint sources, t-f largely joint sources and t-f completely joint sources, but also can classify the closely-spaced sources. The proposed algorithm owns a good DOA estimation performance for all kinds of LFM signals.

\subsection{Summary}

The simulation results explain that the STFT-FBSSMUSIC algorithm can achieve accurate DOA estimation for all kinds of LFM sources. The proposed algorithm can estimate the DOAs of multiple LFM sources accurately by 


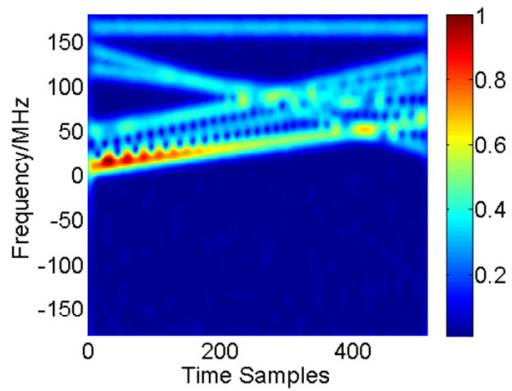

(a)

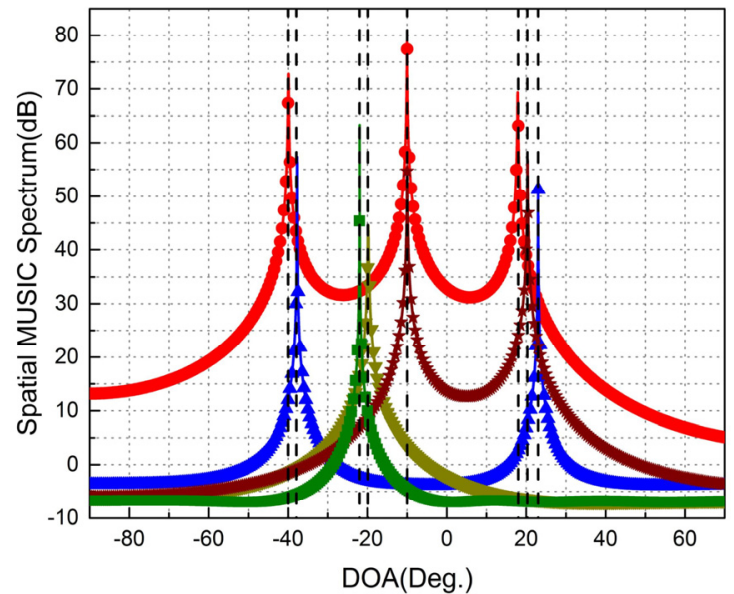

(b)

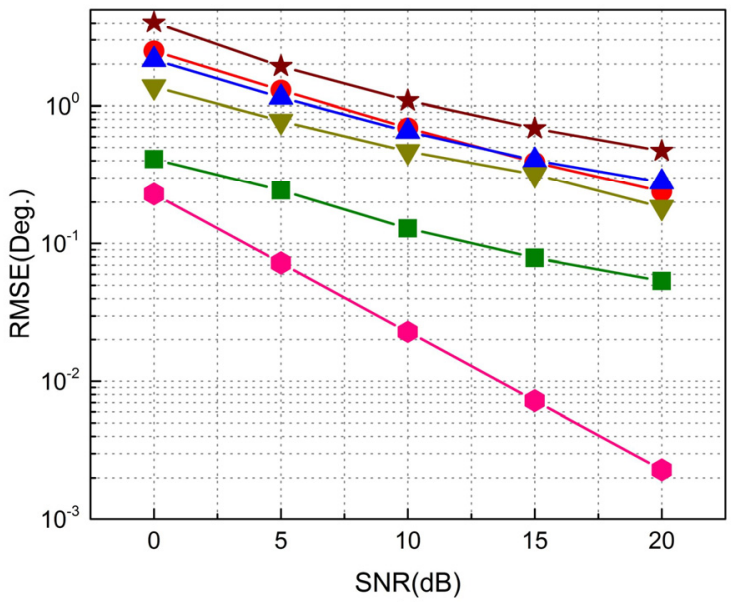

(c)

t-f completely joint sources

-Multiple-source $t-f$ points of $t-f$ tiny joint sources $-\nabla-$ Single-source $t-f$ points of t-f tiny joint sources

$-\star$-Multiple-source $t-f$ points of $t-f$ largely joint sources

$-\square-$ Single-source $t-f$ points of $t-f$ disjoint source

- CRLB

Fig. 9. The estimation results of multiple LFM sources by using the proposed algorithm. (a) The STFT of the LFM sources at $10 \mathrm{~dB}$ SNR. (b) The spatial MUSIC spectrum at $10 \mathrm{~dB}$ SNR. (c) The estimated RMSE versus different SNR levels.

selecting the multiple-source t-f points if the sources are t$f$ largely joint or completely joint in the t-f plane and the single-source $t-f$ points are difficult to be obtained or cannot be obtained. If the LFM sources are t-f tiny joint, the proposed algorithm also can select the multiple-source t-f points to estimate the DOAs. Although the estimation accuracy is lower than that of the algorithm selecting the single-source t-f points, the computational complexity of the proposed algorithm also will be reduced. If the algorithm selects the multiple-source t-f points with $M$ LFM sources, only one time feature decomposition and MUSIC spectrum search need to be carried. In contrast, if we select the single-source t-f points, $M$ times feature decomposition and MUSIC spectrum search need to be carried out. In the case of t-f tiny joint or t-f disjoint LFM sources, the proposed algorithm also can select the single-source t-f points to estimate DOAs of the sources, however, the estimation accuracy is lower than that of the STFT-MUSIC algorithm [35]. This is because the FBSS technique uses the dimension decrease algorithm to solve the problem of rank loss in the STFD matrix caused by the multiple-source t- $f$ points, which brings about the loss of the array aperture and the declines in the estimation performance of the algorithm.

As can be seen from the DOA simulation results of the STFT, SPWVD and FRFT, the estimation performance of the FRFT-based algorithms [10], [13], [15], [16], [32] is poor. Especially in the case that the LFM sources are t-f completely joint, the FRFT-FBSS-MUSIC algorithm even cannot work. In the simulations, we choose the SPWVD for DOA estimation in order to eliminate the influence of the cross-terms generated by the quadratic $t-f$ analysis tools as much as possible. However, the accuracy of the SPWVD is lower than that of the original WVD, which makes the accuracy of the TF-based algorithms [18-20] is less than that of the STFT-based algorithms. The STFTbased algorithms effectively eliminate the influence of the cross-terms and its computational complexity is pretty low. Table 1 shows the computational complexity of several algorithms in the above simulations. In Tab. $1, K$ denotes the sum of the times selecting the single-source t-f points and multiple-source t-f points. If the t-f points are all single-source t-f points, $K=M$. If there are multiple-source t-f points in the selected t-f points, $K<M$. For the $M$ incident sources, the more the number of sources included in the multiple-source $\mathrm{t}-\mathrm{f}$ points is, the smaller the value of $K$ is. The more the times of selecting the multiple-source t- $f$ points is, the smaller the value of $K$ is. As can be seen from the table, the FRFT-based algorithms own the lowest computational complexity, followed by the STFT-based algorithms and the TF-based algorithms. In addition, the computational complexity of the proposed STFT-FBSSMUSIC algorithm is lower than that of the STFT-MUSIC algorithm.

\begin{tabular}{|c|c|}
\hline Algorithm & The computation complexity \\
\hline STFT-MUSIC & $O\left(M N L^{2} \log _{2} L\right)$ \\
\hline STFT-FBSS-MUSIC & $O\left(K k L^{2} \log _{2} L\right)$ \\
\hline TF-MUSIC & $O\left(M N^{2} L^{2} \log _{2} L\right)$ \\
\hline TF-FBSS-MUSIC & $O\left(K k^{2} L^{2} \log _{2} L\right)$ \\
\hline FRFT-MUSIC & $O\left(N L+M N^{2}\right)$ \\
\hline FRFT-FBSS-MUSIC & $O\left(k L+M k^{2}\right)$ \\
\hline
\end{tabular}

Tab. 1. Comparison of the computational complexity. 


\section{Experimental Verification}

We carry out the experiment in the standard microwave anechoic chamber and the sketch map of the experimental platform is shown in Fig. 10. We use two signal generators to produce two far-field sources. The eight channels digital receiver receives the sources from the ULA and stores the signals into the data collector, then, the computer can use the STFT-FBSS-MUSIC algorithm to estimate the DOAs of the sources. In the standard microwave anechoic chamber, the turntable is controlled by the

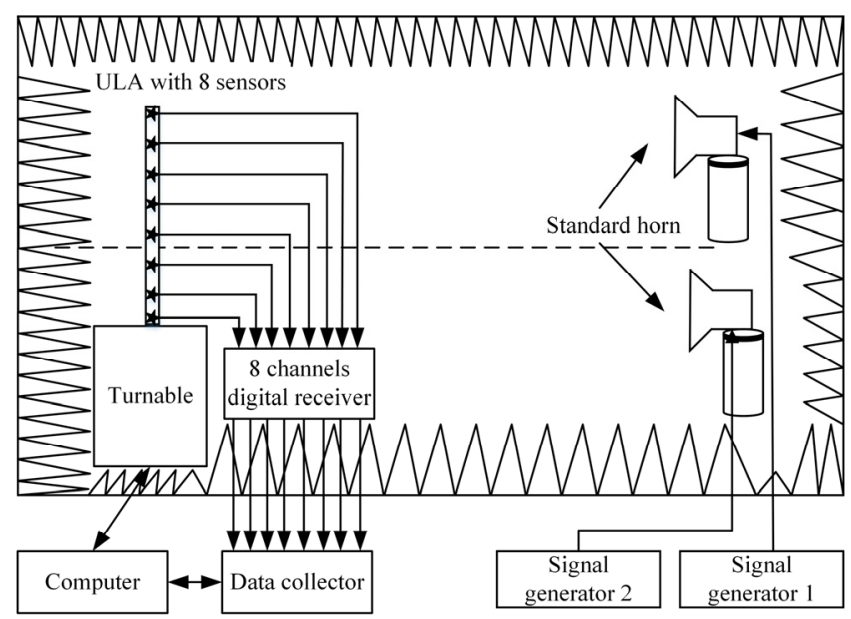

Fig. 10. The sketch map of the experimental platform.

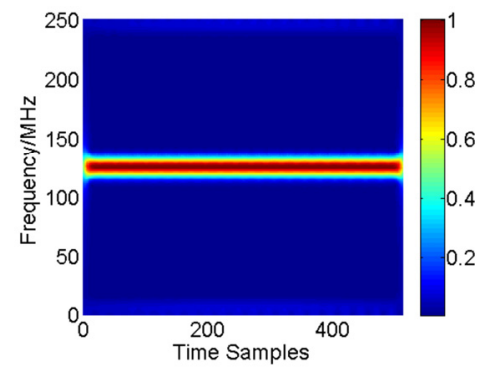

(a)

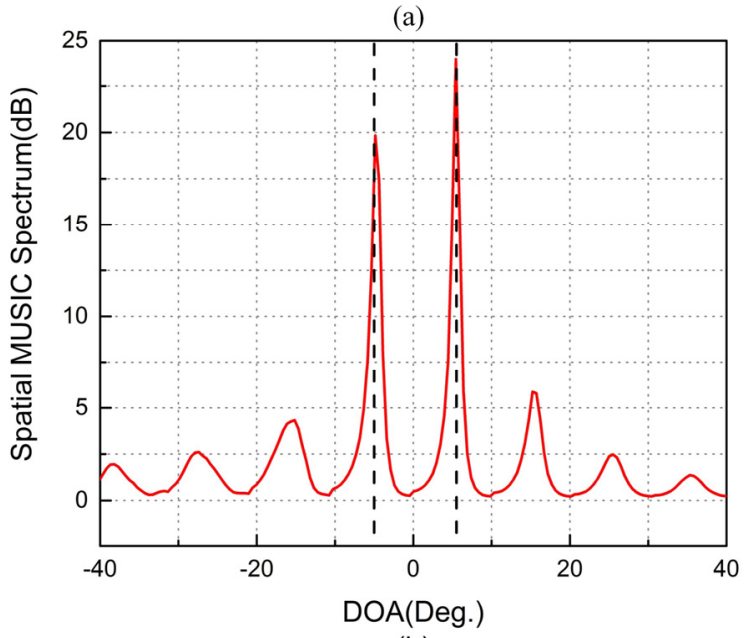

(b)

Fig. 11. The experimental results using the proposed algorithm (a) The STFT of the sources. (b) The spatial MUSIC spectrum. computer and the distance between the horn and the turntable is $17 \mathrm{~m}$. The standard horns are respectively located at $-5^{\circ}$ and $5.5^{\circ}$ relative to the ULA.

Because of the conditions, our signal generators just can produce the single frequency pulse, so we use the two signal generators to produce two signals whose frequency are $3.0 \mathrm{GHz}$ and $3.0 \mathrm{GHz}$, respectively. The amplitude of the signals is $0 \mathrm{dbm}$. The local frequency of the eight channels digital receiver is $2.875 \mathrm{GHz}$ and the sampling frequency of the data collector is $250 \mathrm{MHz}$. The experimental results of the STFT-FBSS-MUSIC algorithm are shown in Fig. 11. As can be seen from Fig. 11(a), the sources are the $\mathrm{t}-\mathrm{f}$ completely joint sources, which means the STFT-FBSSMUSIC algorithm is suitable for estimating the DOAs of these two sources. The spatial MUSIC spectrum (see Fig. 11(b)) indicates that there are two sources because we can see two sharp peaks around the correct DOAs. Through the spectral peak search, we can obtain that the DOA estimations are $-4.86^{\circ}$ and $5.43^{\circ}$, which are in accordance with the sets. The experimental results show that the proposed algorithm can estimate DOAs of the t-f joint sources correctly and has a broad application prospect.

\section{Conclusion}

In order to improve the accuracy of the DOA estimation and eliminate the influence of the cross-terms as much as possible, the existing t-f based DOA estimation algorithms generally need to select the auto-term location of the sources [18], [20], [25], [27], [30] and the single-source t-f points [26], [28], [29], [35], however, if the incident LFM sources are t-f largely joint or completely joint (coherent), the single-source t-f points are very difficult to be selected or cannot be selected, which makes it more difficult for estimating the DOAs of these LFM sources. In order to relax these limitations, this paper presents the STFT-FBSSMUSIC algorithm based on the FBSS technique [37]. The proposed algorithm uses the STFT as the t-f analysis tool, which effectively prevents the influence of the cross-terms and owns a pretty low computational complexity [35]. The proposed algorithm can work in the case of selecting the single-source t-f points or multiple-source t-f points. When selecting the multiple-source t-f points, the estimation accuracy will be reduced slightly, however, selecting the multiple-source t-f points can effectively reduce the times of feature decomposition and MUSIC spectral search, which can reduce the computational complexity. If the LFM sources are t-f largely joint or completely joint, the proposed algorithm can estimate the DOAs by selecting the multiple-source $t-f$ points and using the FBSS technique to solve the problem of rank loss in the STFD matrix caused by the multiple-source $t-f$ points. The simulation results and experimental results show the effectiveness and robustness of the proposed STFT-FBSS-MUSIC algorithm. It can deal with closely-spaced sources and can be applied to all kinds of LFM sources, so it has a high application value. 


\section{References}

[1] WU, Y. T., AMIR, L., JENSEN, J. R., et al. Joint pitch and DOA estimation using the ESPRIT method. IEEE Transactions on Audio, Speech and Language Processing, 2015, vol. 23, no. 1, p. 32-45. DOI: 10.1109/TASLP.2014.2367817

[2] CHEN, H., WAN, Q., FAN, R., et al. Direction-of-arrival estimation based on sparse recovery with second-order statistics. Radioengineering, 2015, vol. 24, no. 1, p. 208-213. DOI: $10.13164 /$ re.2015.0208

[3] PAN, Y. J., TAI, N., YUAN, N. C. Wideband DOA estimation via sparse Bayesian learning over a Khatri-Rao dictionary. Radioengineering, 2015, vol. 24, no. 2, p. 552-557. DOI: $10.13164 /$ re.2015.0552

[4] WANG, L., ZHAO, L. F., BI, G. A., et al. Novel wideband DOA estimation based on sparse Bayesian learning with Dirichlet process priors. IEEE Transactions on Signal Processing, 2016, vol. 64 , no. 2 , p. 275-289. DOI: 10.1109/TSP.2015.2481790

[5] CUI, K. B., WU, W. W., HUANG, J. J., et al. 2D DOA estimation of UCA correlative interferometer based on one dimensional sorting lookup table-two dimensional linear interpolation algorithm. Radioengineering, 2017, vol. 26, no. 2, p. 562-572. DOI: $10.13164 /$ re.2017.0562

[6] SCHMIDT, R. O. Multiple emitter location and signal parameter estimation. IEEE Transactions on Antennas and Propagation, 1986, vol. 34, no. 3, p. 276-280. DOI: 10.1109/TAP.1986.1143830

[7] ROY, R., KAILATH, T. ESPRIT-a subspace rotation approach to estimation of parameters of cissoids in noise. IEEE Transactions on Acoustics, Speech, and Signal Processing, 1986, vol. 34, no. 5 , p. 1340-1342. DOI: 10.1109/TASSP.1986.1164935

[8] ROY, R., KAILATH, T. ESPRIT-estimation of signal parameters via rotational invariance techniques. IEEE Transactions on Acoustics, Speech, and Signal Processing, 1989, vol. 37, no. 7, p. 984-995. DOI: $10.1109 / 29.32276$

[9] GERSHMAN, A. B., AMIN, M. G. Wideband direction-of-arrival estimation of multiple chirp signals using spatial time-frequency distributions. IEEE Signal Processing Letters, 2000, vol. 7, no. 6, p. 152-155. DOI: $10.1109 / 97.844636$

[10] QU, H. T., WANG, R. H., QU, W., et al. Research on DOA estimation of multi-component LFM signals based on FRFT. Wireless Sensor Network, 2009, vol. 3, p. 171-181. DOI: 10.4236/wsn.2009.13023

[11] HEIDENREICH, P., CIRILlO, L. A., ZOUBIR, A. M. Morphological image processing for FM source detection and localization. Signal Processing, 2009, vol. 89, p. 1070-1080. DOI: 10.1016/j.sigpro.2008.12.011

[12] YUAN, X. Direction-finding wideband linear FM sources with triangular arrays. IEEE Transactions on Aerospace and Electronic Systems, 2012, vol. 48, no. 3, p. 2416-2425. DOI: 10.1109/TAES.2012.6237600

[13] CUI, Y., WANG, J. F. Wideband LFM interference suppression based on fractional Fourier transform and projection techniques. Circuits, Systems, and Signal Processing, 2014, vol. 33, no. 2, p. 613 - 627. DOI: $10.1007 / \mathrm{s} 00034-013-9642-\mathrm{z}$

[14] ZHANG, H. J., BI, G. A., YANG, W., et al. IF estimation of FM signals based on time-frequency image. IEEE Transactions on Aerospace and Electronic Systems, 2015, vol. 51, no. 1, p. 326 to 343. DOI: $10.1109 /$ TAES.2014.130554

[15] YU, J. X., ZHANG, L., LIU, K. H. Coherently distributed wideband LFM source localization. IEEE Signal Processing Letters, 2015, vol. 22, no. 4, p. 504-508. DOI: 10.1109/LSP.2014.2363843
[16] YU, J. X., ZHANG, L., LIU, K. H., et al. Separation and localization of multiple distributed wideband chirps using the fractional Fourier transform. EURASIP Journal on Wireless Communications and Networking, 2015, vol. 266, p. 1-8. DOI: 10.1186/s13638-015-0497-9

[17] CUI, K. B., WU, W. W., HUANG, J. J., et al. 2-D DOA estimation of LFM signals based on dechirping algorithm and uniform circle array. Radioengineering, 2017, vol. 26, no. 1, p. 299-308. DOI: 10.13164/re.2017.0299

[18] BELOUCHRANI, A., AMIN, M. G. Time frequency MUSIC. IEEE Signal Processing Letters, 1999, vol. 6, no. 5, p. 109-110. DOI: $10.1109 / 97.755429$

[19] AMIN, M. G., ZHANG, Y. M. Direction finding based on spatial time-frequency distribution matrices. Digital Signal Processing, 2000, vol. 10, p. 325-339. DOI: 10.1006/dspr.2000.0374

[20] ZHANG, Y. M., MU, W. F., AMIN, M. G. Subspace analysis of spatial time-frequency distribution matrices. IEEE Transactions on Signal Processing, 2001, vol. 49, no. 4, p. 747-759. DOI: $10.1109 / 78.912919$

[21] ZHANG, Y. M., AMIN, M. G., HIMED, B. Joint DOD/DOA estimation in MIMO radar exploiting time-frequency signal representations. EURASIP Journal on Advances in Signal Processing, 2012, vol. 102, p. 1-10. DOI: 10.1186/1687-61802012-102

[22] KHODJA, M., BELOUCHRANI, A., ABED-MERAIM, K. Performance analysis for time-frequency MUSIC algorithm in presence of both additive noise and array calibration errors. EURASIP Journal on Advances in Signal Processing, 2012, vol. 94 , p. 1-11. DOI: 10.1186/1687-6180-2012-94

[23] GHOFRANI, S. Matching pursuit for direction of arrival estimation in the presence of Gaussian noise and impulsive noise. IET Signal Processing, 2014, vol. 8, no. 5, p. 540-551. DOI: 10.1049/IET-SPR.2013.0286

[24] LIN, J. C., MA, X. C., YAN, S. F., et al. Time-frequency multiinvariance ESPRIT algorithm for DOA estimation. IEEE Antennas and Wireless Propagation Letters, 2016, vol. 15, p. 770-773. DOI: 10.1109/LAWP.2015.2473664

[25] LINH-TRUNG, N., BELOUCHRANI, A., ABED-MERAIM, K., et al. Separating more sources than sensors using time-frequency distributions. EURASIP Journal on Applied Signal Processing, 2005, vol. 17, p. 2828-2847. DOI: 10.1155/ASP.2005.2828

[26] ZHANG, Y. M., AMIN, M. G. Blind separation of nonstationary sources based on spatial time-frequency distributions. EURASIP Journal on Applied Signal Processing, 2006, p. 1-13. DOI: 10.1155/ASP/2006/64785

[27] BELOUCHRANI, A., AMIN, M. G. Blind source separation based on time-frequency signal representations. IEEE Transactions on Signal Processing, 1998, vol. 46, no. 11, p. 2888-2897. DOI: $10.1109 / 78.726803$

[28] YILMAZ, O., RICKARD, S. Blind separation of speech mixtures via time-frequency masking. IEEE Transactions on Signal Processing, 2004, vol. 52, no. 7, p. 1830-1847. DOI: 10.1109/TSP.2004. 828896

[29] BARKAT, B., ABED-MERAIM, K. Algorithms for blind components separation and extraction from the time-frequency distribution of their mixture. EURASIP Journal on Applied Signal Processing, 2004, vol. 13, p. 2025-2033. DOI: $10.1155 / \mathrm{S} 1110865704404193$

[30] AÏSSA-EL-BEY, A., LINH-TRUNG, N.,, ABED-MERAIM, K., et al. Underdetermined blind separation of nondisjoint sources in the time-frequency domain. IEEE Transactions on Signal Processing, 2007, vol. 55, no. 3, p. 897-907. DOI: $10.1109 /$ tsp.2006.888877

[31] FADAILI, E. M., MOREAU, N. T., MOREAU, E. Nonorthogonal joint diagonalization/zero diagonalization for source separation 
based on time-frequency distributions. IEEE Transactions on Signal Processing, 2007, vol. 55, no. 5, p. 1673-1687. DOI: 10.1109/TSP. 2006.889469

[32] YANG, W., SHI, Y. W. FRFT based method to estimate DOA for wideband signal. Advanced Materials Research, 2015, vol. 712715, p. $2716-2720$. DOI: 10.4028 /www.scientific.net/AMR.712715.2716

[33] CUI, K. B., WU, W. W., HUANG, J. J., et al. DOA estimation of LFM signals based on STFT and multiple invariance ESPRIT. International Journal of Electronics and Communications, 2017, vol. 77, p. 10-17. DOI: 10.1016/J.AEUE.2017.04.021

[34] ZHANG, Y. M., AMIN, M. G., HIMED, B. Direction-of-arrival estimation of nonstationary signals exploiting signal characteristics. In 11th International Conference on Information Science, Signal Processing and Their Applications. Montreal (Canada), 2012, p. 1223-1227. DOI: 10.1109/ISSPA.2012.6310479

[35] ZHANG, H. J., BI, G. A., CAI, Y. L., et al. DOA estimation of closely-spaced and spectrally-overlapped sources using a STFTbased MUSIC algorithm. Digital Signal Processing, 2016, vol. 52, p. 25-34. DOI: 10.1016/j.dsp.2016.01.015

[36] SHAN, T. J., WAX, M., KAILATH, T. On spatial smoothing for direction-of-arrival estimation of coherent signals. IEEE Transactions on Acoustics, Speech, and Signal Processing, 1985, vol. 33, no. 4, p. 806-811. DOI: 10.1109/TASSP.1985.1164649

[37] PILLAI, S. U., KWON, B. H. Forward/backward spatial smoothing techniques for coherent signal identification. IEEE Transactions on Acoustics, Speech, and Signal Processing, 1989, vol. 37, no. 1, p. 8-15. DOI: 10.1109/29.17496

[38] LIU, F. L., WANG, J. K., SUN, C. Y., et al. Spatial differencing method for DOA estimation under the coexistence of both uncorrelated and coherent signals. IEEE Transactions on Antennas and Propagation, 2012, vol. 60, no. 4, p. 2052-2062. DOI: 10.1109/TAP.2012.2186216

[39] MA, X. R., DONG, X. H., XIE, Y. F. An improved spatial differencing method for DOA estimation with the coexistence of uncorrelated and coherent signals. IEEE Sensors Journal, 2016, vol. 16, no. 10, p. 3719-3723. DOI: 10.1109/JSEN.2016.2532929

[40] JOURJINE, A., RICKARD, S., YILMAZ, O. Blind separation of disjoint orthogonal signals: demixing $\mathrm{N}$ sources from 2 mixtures. In IEEE International Conference on Acoustics, Speech, and Signal Processing. Istanbul (Turkey), 2002, vol. 5, p. 2985-2988. DOI: 10.1109/ICASSP.2000.861162

[41] BELOUCHRANI, A., ABED-MERAIM, K., AMIN, M. G., et al. Joint anti-diagonalization for blind source separation. In IEEE International Conference on Acoustics, Speech, and Signal
Processing. Salt Lake City (USA), 2001, vol. 5, p. 2789-2792. DOI: 10.1109/ICASSP. 2001.940225

[42] CHEN, Y. M., LEE, J. H., YEH, C. C., et al. Bearing estimation without calibration for randomly perturbed arrays. IEEE Transactions on Signal Processing, 1991, vol. 39, no. 1, p. 194-197. DOI: $10.1109 / 78.80780$

[43] LINEBARGER, D. A. Redundancy averaging with large arrays. IEEE Transactions on Signal Processing, 1993, vol. 41, no. 4, p. $1707-1710$. DOI: $10.1109 / 78.212750$

[44] STOICA, P., NEHORAI, A. MUSIC, maximum likelihood, and Cramer-Rao bound. IEEE Transactions on Acoustics, Speech and Signal Processing, 1989, vol. 37, no. 5, p. 720-741. DOI: $10.1109 / 29.17564$

[45] CHAMBers, C., TOZER, T. C., SHARMAN, K. C., et al. Temporal and spatial sampling influence on the estimates of superimposed narrowband signals: when less can mean more. IEEE Transactions on Signal Processing, 1996, vol. 44, no. 12, p. 3085-3098. DOI: $10.1109 / 78.553482$

\section{About the Authors ...}

Kaibo CUI was born in 1990. He received his M.S. degree in Electronic Science and Technology from the National University of Defense Technology in 2013. His research interests include signal processing and spatial spectrum estimation.

Xi CHEN was born in 1983. He received his Ph.D. degree in Electronic Science and Technology from the National University of Defense Technology in 2013. His research interests include signal processing.

Jingjian HUANG was born in 1983. He received his Ph.D. degree in Electronic Science and Technology from the National University of Defense Technology in 2014. His research interests include antenna design.

Naichang YUAN was born in 1965. He received his M.S. and Ph.D. degree in Electronic Science and Technology from the University Science and Technology of China in 1991 and 1994, respectively. His research interests include array signal processing, signal processing in radar. 